\title{
Roll Damping Derivatives from Generalized Lifting-Surface Theory and Wind Tunnel Forced-Oscillation Tests
}

\author{
Anthony S. Pototzky ${ }^{1}$ and Patrick C. Murphy ${ }^{2}$ \\ NASA Langley Research Center, Hampton, VA, 23681-2199
}

\begin{abstract}
Improving aerodynamic models for adverse loss-of-control conditions in flight is an area being researched under the NASA Aviation Safety Program. Aerodynamic models appropriate for loss of control conditions require a more general mathematical representation to predict nonlinear unsteady behaviors. As more general aerodynamic models are studied that include nonlinear higher order effects, the possibility of measurements that confound aerodynamic and structural responses are probable. In this study an initial step is taken to look at including structural flexibility in analysis of rigidbody forced-oscillation testing that accounts for dynamic rig, sting and balance flexibility. Because of the significant testing required and associated costs in a general study, it makes sense to capitalize on low cost analytical methods where possible, especially where structural flexibility can be accounted for by a low cost method. This paper provides an initial look at using linear lifting surface theory applied to rigid-body aircraft roll forced-oscillation tests.
\end{abstract}

\section{Nomenclature}

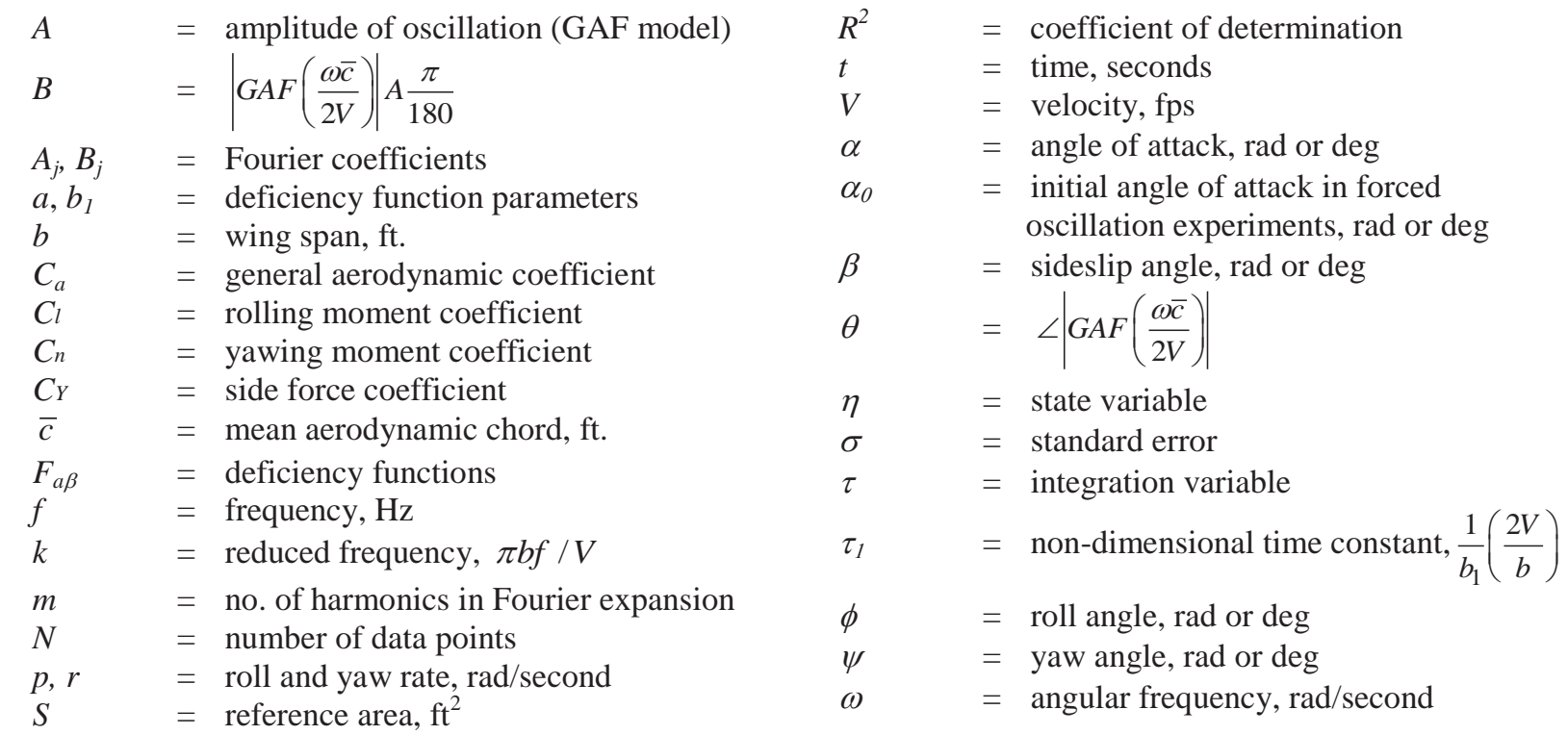

\section{Introduction}

$\mathrm{T}$ The NASA Aviation Safety Program supports development of systems for improved safety of flight. These systems allow recovery from or prevention of entry into loss of control conditions, one of the largest contributors to fatal aircraft accidents. ${ }^{1}$ One element of this research is development of high fidelity aerodynamic models for transport aircraft over an extended flight envelope including regions where nonlinear and unsteady

\footnotetext{
${ }^{1}$ Senior Research Engineer, Aeroelasticity Branch, Mail Stop 340, Senior Member.

${ }^{2}$ Senior Research Engineer, Dynamic Systems and Control Branch, Mail Stop 308, Associate Fellow.
} 
behaviors may occur. ${ }^{2-6}$ Although these studies have focused primarily on damping, nonlinear, and unsteady behaviors for rigid-body aircraft, observations during dynamic testing indicate the likely presence of some structural responses. Possible sources of structural flexibility are from the dynamic test rig, sting, balance, and the test article. It is common practice to assume these structural responses occur at frequencies well above any modes of interest in the damping studies. As more general aerodynamic models are studied that include nonlinear unsteady aerodynamics with higher order modes, the likelihood of obtaining measurements that confound rigid-body aerodynamics and structural responses increases. This type of modeling work places significant demand on resources for both analytical computations using high fidelity codes and experimental testing to obtain static and dynamic data. Because of the significant testing required and associated costs, it makes sense to capitalize on low cost analytical methods where possible, especially where structural flexibility can be accounted for by these less costly methods. With the appropriate assumptions of linearity, methods such as doublet lattice can provide damping and unsteady model parameters very efficiently, with reasonable accuracy and at low cost.

As a first step toward investigating the utility of doublet lattice, this paper will show that simple linear lifting surface theory can produce unsteady-generalized aerodynamic force (GAF) coefficients comparable to experimental measurements. The more general question of when nonlinear dynamics are confounded with structural modes will be addressed in future studies. The lifting surface theory in MSC NASTRAN uses the doublet lattice method ${ }^{7}$ to compute these unsteady GAF coefficients from which dynamic stability derivatives can be obtained in the form of conventional in-phase and out-of-phase coefficients. In this form, comparisons can conveniently be made with corresponding wind tunnel results. Partially to demonstrate the utility and effectiveness of these analytically generated coefficients, the paper will additionally show an analytical model of the wind-tunnel test setup. This task was done by building a finite-element, structural-dynamics model of the rotating mounting system to the earlier developed analytical rigid-body unsteady aerodynamic model generated by doublet lattice. This finite-element modeling of the wind-tunnel model with the support structure, which was used to obtain stability derivative data, will be discussed and presented in the paper. As part of this exercise, the paper will show simulation results of structural force and moment time-histories produced by MSC NASTRAN finite-element analysis (FEA) similar to the force and moment signal data obtained from the model balance during wind-tunnel testing.

\section{Modeling}

Aerodynamic models in this study are designed to represent one-degree of freedom roll forced-oscillation experiments. Computational and experimental models are designed to capture roll aerodynamics for a rigid-body aircraft. The computational model includes structural flexibility introduced by the dynamic rig, sting, and balance.

Wind tunnel tests were conducted in 2009 at the NASA Langley 14x22 Wind Tunnel. The NASA Generic Transport Model (GTM), a 5.5\% scale model representing a conventional twin-engine commercial transport, was tested. A variety of amplitude and frequencies were tested over a large range of angle of attack and sideslip. Figure 1 shows the experimental setup.

\section{A. Computational Model}

The first task of producing GAFs requires the application of NASTRAN, which provides the Finite Element Analysis (FEA) and unsteady aerodynamic analysis, and a code internal to NASTRAN, DMAP ${ }^{8}$ (Direct Matrix Abstraction Program), which generated the

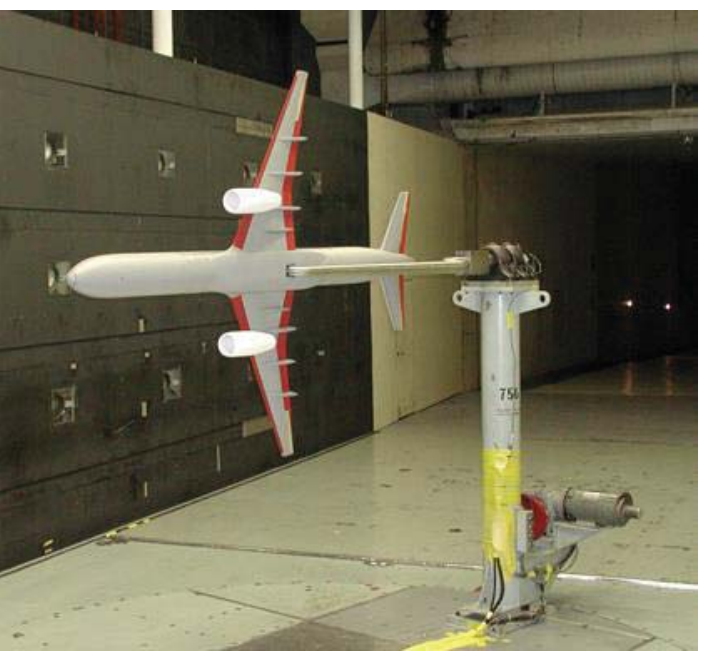

Figure 1. The GTM wind-tunnel model sting mounted on the roll-oscillation rig inside NASA Langley's 14x22 Wind Tunnel. prescribed rigid-body mode-shapes and outputted the GAF coefficients. The second task to produce wind-tunnel model simulation results requires only a straightforward application of solution 146, which is NASTRAN's Aeroelastic Response Analysis. Composing the analytical wind-tunnel model required additional information, such as the physical composition of the wind-tunnel model, mounting system hardware and the aerodynamic balance.

Because of the relative simplicity of generating the GAF coefficients using doublet lattice/lifting surface theory, ${ }^{9}$ its utility can be expanded beyond modeling the experimental wind-tunnel test setup as demonstrated here in the 
paper. This method can easily be used to generate coefficients for "damaged" aircraft configurations such as missing engines or partially destroyed/damaged wing/tail surfaces. Even for undamaged aircraft configurations, this method has utility in generating coefficients for control surface modes such as horizontal tail, elevators, flaps, ailerons, rudders and so on. Especially if these surfaces have inherent unsteady dynamic behaviors, this method is a good choice for generating unsteady coefficients when only linear aerodynamics is warranted to model dynamic stability derivatives. This method, at minimum, can give an initial assessment of unsteady aerodynamic effects on stability derivatives.

Doublet lattice and NASTRAN FEA have several potential advantages besides low cost and speed. The code can be used to explore the effects of very-low speed, transonic or supersonic flow conditions, model mass unbalance, and aeroelastic responses of flexible aircraft and, as presented here, the aeroelastic effects of wind-tunnel models attached to a dynamic rig with a flexible sting structure, an aerodynamic loads balance, and while undergoing forced oscillations.

Since doublet lattice is based on potential theory, the results are only appropriate for low angles of attack with attached flows and linear aerodynamics. Comparisons in this study will identify the allowable ranges of angle of attack, amplitude, and frequency that maintain linear aerodynamic responses and valid comparisons of the two data sources.

1. Aerodynamic and FE Structural Dynamics Model

To develop these analytical models, MSC PATRAN with the FLIGHT LOADS preference was used to put together the doublet lattice aerodynamic modeling and the finite-element structural modeling of both the windtunnel model and the test-rig structure for mounting the aircraft configuration. Most of the initial effort involved using the PATRAN GUI capability that generates, handles and performs any geometric processing needed to derive a suitable aerodynamic geometry to best represent the GTM geometry into a more simplified flat-plate or lifting surface representation needed to generate doublet-lattice unsteady aerodynamics. Next, FLIGHT LOADS, which is a preference or a "module" within PATRAN, was used to generate, handle and process the aeroelastic entities needed as input for NASTRAN code to run an aeroelastic analysis. Specifically, FLIGHT LOADS is used to generate the aerodynamic surface grids or "boxes," the spline surfaces, and the "coupling" of the spline surfaces and the aerodynamic grids to the finite-element structure. In the final stage of modeling, PATRAN was used to develop and process the structural parts or entities for the NASTRAN finite elements code. Here PATRAN is utilized for its primary function to be the pre-processor, and post-processor, to NASTRAN finite-element analysis capability.

2. Structural and Spline Surfaces for the GTM Aircraft Configuration

No structural or aeroelastic work with NASTRAN analysis has been performed on the GTM wind-tunnel model and test setup prior to this paper. Consequently, the aeroelastic modeling began using an IGES (Initial Graphics Exchange Specification) formatted geometry file available from the analysis efforts performed with the USM-3D CFD code ${ }^{3}$, as is shown in Fig. 2. The geometry depicted in Fig. 2 accurately reflects both dimensions and the outer-moldline shape of the wind-tunnel configuration so the dimensional numbers and the aerodynamic shape were carefully followed for the modeling effort of this paper. PATRAN with its graphical utility was especially useful for handling this type of geometry and was used to develop the flatplate representation of the entire GTM aircraft configuration needed for the unsteady aerodynamic computations with the doublet lattice code. This type of representation

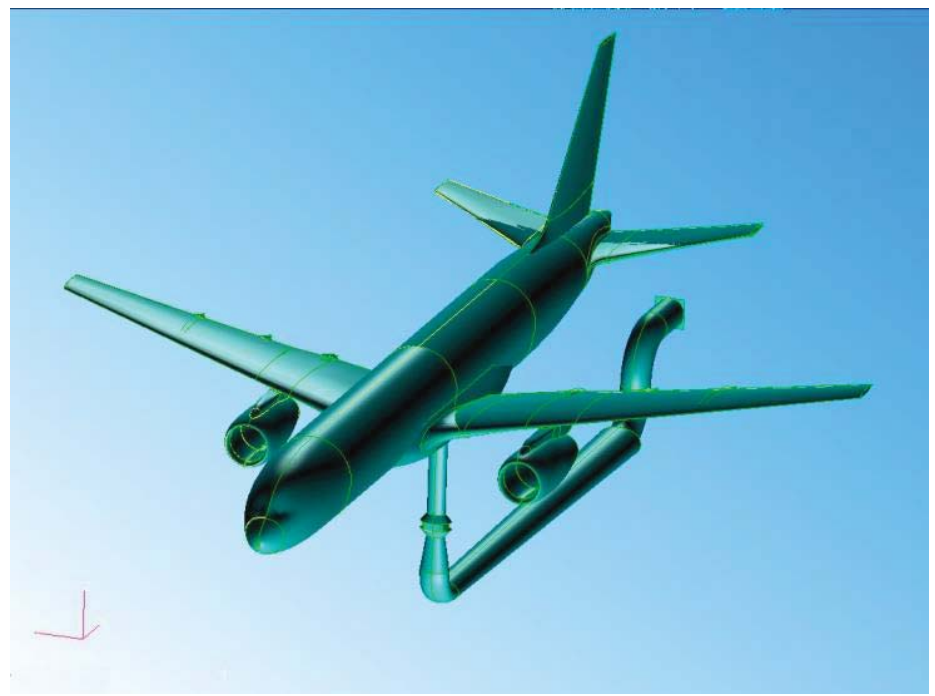

Figure 2. GTM geometry used to produce aerodynamic results with the USM-3D CFD (computational fluid dynamics) code. is typically used in transport configuration modeling. This approach includes remodeling engines and the fuselage as cruciform-shaped flatplate surfaces. Conventionally, the strategy for using such cruciform flat surfaces in this fashion is to be able to easily add aerodynamic correction factors coming from wind-tunnel testing or from CFD to more accurately model the 
aerodynamics later in the design process. The vertical part of the cruciform along the centerline of the fuselage provide the aerodynamic forces in the lateral direction, which extends from the aircraft's roof, down to its keel and crosses the "floorboard" part of the cruciform.

Forming the wing and engine aerodynamic surfaces had its own unique set of geometric requirements. The approach used to form the appropriate outward inclination of the configuration's wing and horizontal tail surfaces was to use their respective leading edges in the geometry presented in Fig. 2 as a guide for their dihedral angles. To form the wing- and tail-plane surfaces, it was assumed in the windstream direction that these surfaces would have no elevation changes from the leading edge to the trailing edges at a particular span station when building the flat-plate representation as shown in Fig. 3. Ordinarily, wing geometries are designed to produce local lift with positive incident angles at most span stations, but with the doublet lattice theory, these local incident angles are instead produced analytically during the downwash part of the processing of the unsteady aerodynamic computations of the code.

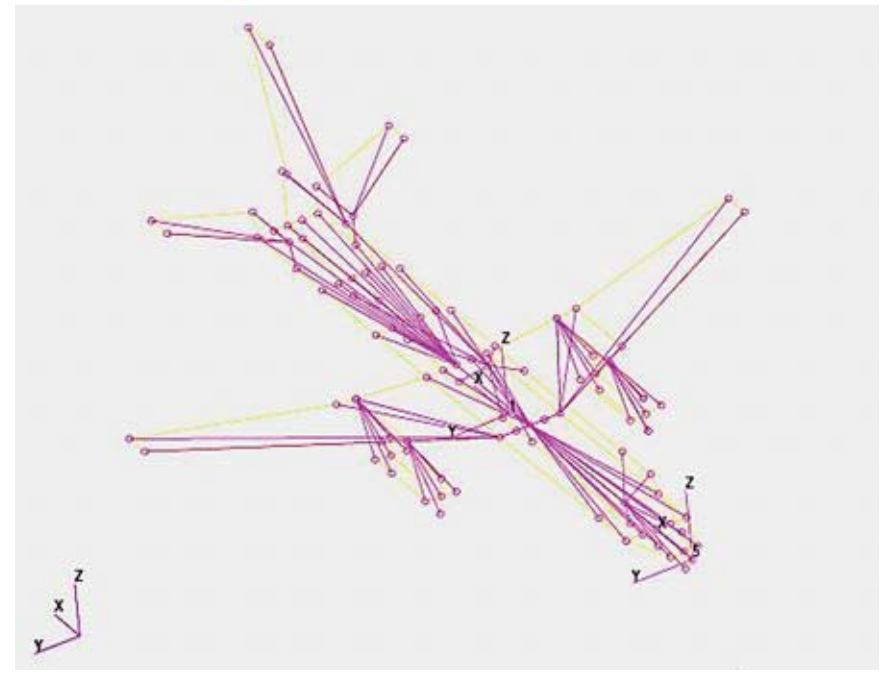

Figure 3. NASTRAN finite element model with rigid elements of the GTM configuration.

In an effort to generate a more realistic lift distribution along the horizontal part of fuselage representation, it was necessary to layout the FEM structure such that it can support a continguous aerodynamic surface from nose to the tail-cone of the aircraft. Using this approach in forming the support structure, the aerodynamic surface produces lift forces that is highest at the leading edges and gradually decreases toward trailing edge part of the surface. The wing carrythough is at the same level as the floorboard aerodynamic surface just beneath the actual aircraft's cabin floor level. Although the horizontal tail carry-through is higher than that of the wing's, the carry-though on the inboard part of the horizontal tail surface was not extended directly into the vertical tail at the aircraft's centerline as was the wing's, rather it changes direction downward and is connected to the outer edge of the fuselage floorboard. Nevertheless, the vertical fin surface is connected directly to the floorboard surface as shown in Fig. 3.

To distinguish all the flatplate surfaces generated with PATRAN and to help generate the structural linkages needed in performing the unsteady aerodynamic computations, PLOTEL bar elements were created with PATRAN to outline their outer edges with yellow lines for each surface as shown in Fig. 3. These flatplate surfaces with the highlighted edges are used to form the individual aerodynamic panels for the doublet lattice computations and the corner points of the highlighted flatplate serve as anchor points where the rigid-element "structures" are attached to the aerodynamic panels. PLOTEL elements are nonstructural and are used here to simply identify the boundaries of the individual flatplate surfaces or the aerodynamic panels; however, later when performing NASTRAN aeroelastic analysis, PLOTEL elements are also used to track the motion of the aerodynamic panels graphically with PATRAN.

Since the wind tunnel model was very light and very stiff, constructing the "structure" with RBE1, a rigid element, was an easy and ideal choice. These rigid elements have the unique property of allowing kinematic motions of the finite-element structure but no elastic motions where the structure is allowed to flex. The spline "surfaces" that are attached to the structure move the aerodynamic surfaces. Generally, when generating aeroelastic surfaces with flexible structures, there are a larger number of structural gridpoints attached to the spline to sufficiently define the flexing of the structural surfaces. Since the panels are rigid, the construction of the structure is much more simplified and only the gridpoints at the corners of the panels need to be attached to the spline to define rigid-body motions.

The purple lines representing the rigid elements in Fig. 3 depict the construction method for a rigid structure. The construction process of the rigid elements started with the mass center point of the wind tunnel model and spreads radially out in the various directions to the aerodynamic panel surfaces of the aircraft configuration. The composition of this rigid structure is analogous to a tree where the various branches spread out from its trunk.

3. Aerodynamic Boxes for Doublet Lattice Unsteady Aero Computations

Setting up models, obtaining analytical results, and performing unsteady computations with the doublet lattice code is a relatively simple process as compared to the steps required to obtain similar results from CFD. For lifting surface theory, at each one of the aerodynamic box locations, the forces applied by the fluid pressure to the structure

4 
are only separated by a half box chord to the downwash location. The flatplate surfaces whose development was described in the previous section are the basis for the aerodynamic panels needed to model unsteady aerodynamics. These flatplate-surface locations are populated by the FLIGHT LOADS preprocessor with the aerodynamic boxes. The boxes created for the doublet lattice NASTRAN aeroelastic computations are shown in Fig. 4. Note that the fuselage and the engines are comprised of boxes on the cruciform surfaces in the horizontal and the vertical directions, which is the customary method of modeling aerodynamic boxes for a transport configuration. The sequence used to generate these aerodynamic boxes on the various surfaces was to define the boxes of the right wing and the engine surfaces first. The boxes of the horizontal and the vertical surfaces of the fuselage were created second, along with the boxes on the vertical fin and the horizontal tails. Finally, the boxes of the left wing and the engine were formed last.

The orientation for the aerodynamic box surfaces is critical and must follow the grid numbering convention of "the right-hand rule." In generating the GTM aerodynamic configuration, aerodynamic modeling of the vertical centerline boxes requires particular care in attempting to obtain the correct box orientation using the FLIGHT LOADS pre-processing software. In the case at hand, the problem with the orientation of this particular set of boxes shows up when attempting to use the "verify" spline option within FLIGHT LOADS. When the spline/aerodynamic surfaces are incorrectly oriented, these spline surfaces deflected in the opposite direction to actual mode shape deflections while the correctly oriented ones deflected in the same direction and track the actual mode shape deflections as they should. After using FLIGHT LOADS to set up these erroneously oriented boxes, it cannot be used to correctly orient them, which is a known deficiency within the code. However, if for some reason any of the boxes are not correctly orientated using FLIGHT LOADS, the NASTRAN code, which usually follows the FLIGHT LOADS processing, has sufficiently comprehensive software in solutions 144, 145 and 146 to re-orient the boxes in the appropriate or proper directions and automatically

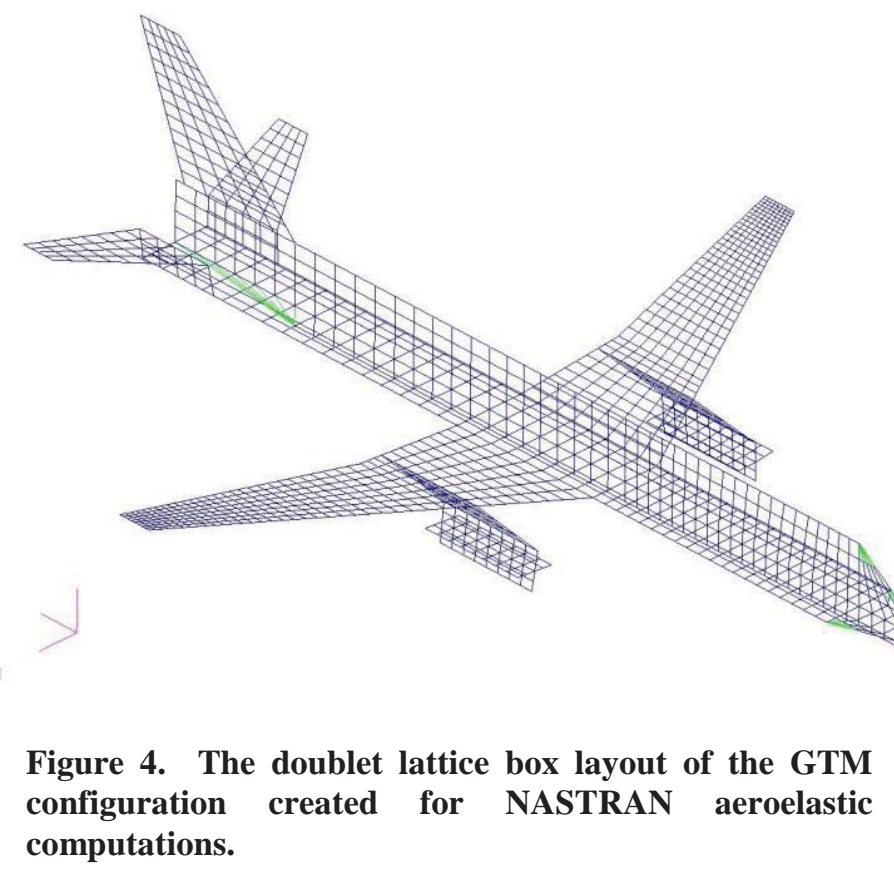
correct the problem.

\section{B. Experimental Model}

Analysis of the wind tunnel dynamic data is accomplished using harmonic analysis ${ }^{10}$ and both sources of dynamic data are analyzed using system identification methods ${ }^{11}$ to estimate unsteady terms when present.

The conventional method to obtain damping coefficients dynamic data experimentally is to perform forcedoscillation tests at various frequencies and amplitudes over the flight envelope of interest. To obtain a more general model that includes any unsteady aerodynamic behaviors a slightly more general model is needed. A simplified model based on indicial functions ${ }^{10}$ has the form shown in Eq. (1). In this equation, $C_{a}$ is a general aerodynamic coefficient representing rolling moment, yawing moment, or side force, that is $a=l, n$ or $Y$ repectively.

$$
C_{a}(t)=C_{a_{\beta}}(\infty) \beta(t)+\frac{b}{2 V} C_{a_{p}}(\infty) p(t)+\frac{b}{2 V} C_{a_{r}}(\infty) r(t)-\int_{0}^{t} F_{a_{\beta}}(t-\tau) \beta(\tau) d \tau
$$

where the steady term, $C_{a}(0)$, has been subtracted from both sides of Eq. (1) to take into account changes with respect to steady state. To obtain a model appropriate for identification and with a limited number of parameters, the deficiency function is assumed to be a simple exponential function, ${ }^{12}$

$$
F_{a_{\beta}}=a e^{-b_{1} t}
$$


Models appropriate for an aircraft undergoing one degree of freedom forced oscillation in roll or yaw can be obtained using Eqs. (1) and (2). Considering one degree of freedom rolling motion in the tunnel,

$$
C_{a}(t)=C_{a}[\phi(t), p(t)]
$$

where roll angle is kinematically related to the sideslip angle by the equation,

$$
\beta(t)=\sin ^{-1}(\sin \alpha \sin \phi(t))
$$

Combining Eqs. (1-4), the aerodynamic models can be formulated as,

$$
C_{a}(t)=C_{a_{\beta}}(\infty) \beta(t)+\frac{b}{2 V} C_{a_{p}}(\infty) p(t)-a \int_{0}^{t} e^{-b_{1}(t-\tau)} \beta(\tau) d \tau
$$

By introducing,

$$
\eta(t)=\int_{0}^{t} e^{-b_{1}(t-\tau)} \beta(\tau) d \tau
$$

and applying the Leibnitz integral rule, the state space form of Eq. (5) can be written as,

$$
\begin{gathered}
\dot{\eta}(t)=-b_{1} \eta(t)+\dot{\beta}(t) \\
C_{a}(t)=C_{a_{\beta}}(\infty) \beta(t)+\frac{b}{2 V} C_{a_{p}}(\infty) p(t)-a \eta(t) .
\end{gathered}
$$

From Eq. (5), a steady response can be obtained ${ }^{12}$ as,

$$
C_{a}(t)=\bar{C}_{a_{\beta}} \phi_{A} \sin (\omega t)+\bar{C}_{a_{p}} \phi_{A} k \cos (\omega t)
$$

where $\phi_{A}$ is the amplitude of roll oscillation; $k$ is reduced frequency; and where $\bar{C}_{a_{\beta}}$ and $\bar{C}_{a_{p}}$ are the in-phase and out-of-phase components, respectively. These components are related to the model parameters by the equations, ${ }^{13}$

$$
\begin{gathered}
\bar{C}_{a_{\beta}}=C_{a_{\beta}}(\infty) \sin \alpha_{0}-a \frac{\tau_{1}^{2} k^{2}}{1+\tau_{1}^{2} k^{2}} \sin \alpha_{0} \\
\bar{C}_{a_{p}}=C_{a_{p}}(\infty)-a \frac{\tau_{1}}{1+\tau_{1}^{2} k^{2}} \sin \alpha_{0} .
\end{gathered}
$$

where $\tau_{1}$ is the non-dimensional inverse of $b_{1}$, given as $\tau_{1}=\frac{1}{b_{1}}\left(\frac{2 V}{b}\right)$.

For this study, a method of harmonic analysis ${ }^{14}$ was applied to measured aerodynamic coefficients. A mathematical model for these coefficients is,

$$
C_{a}(t)=A_{0}+\sum_{j=1}^{m} A_{j} \cos (j \omega t)+\sum_{j=1}^{m} B_{j} \sin (j \omega t) \quad a=l, n \text { or } Y
$$


where $A_{0}, A_{j}$ and $B_{j}$ are the Fourier coefficients. The analysis provides estimates of these coefficients, their standard errors, and the coefficient of determination, $R^{2}$. The coefficient of determination, $R^{2}$, indicates the fraction of variation in the measured data about the mean explained by the model and is defined as,

$$
R^{2}=1-S S_{E} / S S_{r}, \quad 0<R^{2}<1
$$

where,

$$
S S_{E}=\sum_{i=1}^{N}\left[C_{a_{E}}(i)-\hat{C}_{a}(i)\right]^{2}
$$

is the residual sum of squares and

$$
S S_{r}=\sum_{i=1}^{N}\left[C_{a_{E}}(i)-\mathcal{C}_{a}^{0}(i)\right]^{2}
$$

is the total sum of squares. $C_{a_{E}}(i), \hat{C}_{a}(i)$, and $\mathscr{C}_{a}^{0}(i)$ are the measured, estimated, and mean values, respectively.

For the model with linear aerodynamics and $A_{0}=0$, the aerodynamic in-phase and out-of-phase components can be expressed in terms of the coefficients $A_{1}$ and $B_{1}$. For the roll oscillation case, the expressions are,

$$
\begin{gathered}
\bar{C}_{a_{\beta}}=\frac{B_{1}}{\phi_{A}} \\
\bar{C}_{a_{p}}=\frac{A_{1}}{k \phi_{A}}
\end{gathered}
$$

where $\beta$ is related to $\phi$ by Eq. (4).

\section{GAF Coefficients for Rigid-Body Motions}

The NASTRAN aerodynamic and finite-element modeling setup to calculate the unsteady GAF coefficients was a simpler task than the NASTRAN setup required to model the wind-tunnel sting mounting structure to obtain the dynamic roll derivative data. To obtain the 6 degree-of-freedom rigid-body derivatives, a simple two-beam structure was used. Each structure is an inch long and each juts out in opposite directions laterally away from the FEM mass center point, which is also the FEM center of gravity (c.g.). Furthermore, the opposite ends of these beams, away from the c.g. point, were tied to ground. This structural arrangement permits the moment center and the elastic center to be coincidental and connected to the mass center, as well as, to the converged point of all the forces and moments produced by the aerodynamics of the lifting surfaces representing the wind-tunnel (WT) model. In addition, NASTRAN produced with this arrangement six vibration modes with pitch, yaw and roll rotational mode shapes and with plunge, side-slip and fore-aft translational mode shapes. Using DMAP coding with NASTRAN, it was possible to generate prescribed rotational and translational mode-shape with either one-radian rotational or unity translational deflections. Furthermore, these deflections had the appropriate directions assigned to each of the rigidbody mode shapes allowing the associated GAF coefficients to have the appropriate signs and magnitudes after being generated by the doublet lattice computations within NASTRAN.

In the process of generating these GAF coefficients, especially the ones associated with aerodynamic moments, care was taken to use the specified and correct moment center in the NASTRAN's input data. Identifying the correct moment center position is important in producing representative aerodynamic moment results. If the moment center is offset from the correct location, aerodynamic moment results will also be offset. Offset information can easily occur, especially if the results come from two or more different sources such as experimental or analytical studies. For this particular analysis, information that provided this specific location (to ensure the correct and accurately 
representative moment results) came from drawing of the fuselage bulkheads used to setup the wind-tunnel model for the dynamic-stability-derivative testing in the $14 \times 22$ wind tunnel.

NASTRAN's solution 145 (SOL 145), which is the standard solution sequence used for flutter analysis, was also used to obtain the generalized aerodynamic force coefficients for the six degree of freedom rigid-body problem. For completeness, GAFs for a full range of reduced frequencies were calculated which included frequencies near zero, important for the rigid-body motions (up to 0.1), and a range, covering the flexible modes, up to a reduced frequency of 3.0. Also to understand the dynamics and the aerodynamic intermodal coupling, especially at the higher frequencies among the six rigid-body modes, all 36 GAFs were computed including the cross terms between the symmetrical and anti-symmetric configurations, which are ordinarily ignored. Finally, as customary with doublet lattice computations, these GAF coefficient results were not scaled by dynamic pressure.

Most time-history wind-tunnel roll moment results are most conveniently presented as ellipsoid plots, ${ }^{10}$ however, the results available from doublet lattice normally come in the form of frequency-dependent complex GAFs with real and imaginary parts representing the in-phase and out-of-phase components of the coefficients. These coefficients can be represented as a time-dependent sinusoidal response of the $G A F(k)$ using the following sine function representation:

$$
y(t)=B \sin (\omega t+\theta)
$$

The time-dependent sinusoidal input to the $\mathrm{GAF}(\mathrm{k})$ as,

$$
x(t)=A \sin (\omega t)
$$

where $B=\left|G A F\left(\frac{\omega \bar{c}}{2 V}\right)\right| A \frac{\pi}{180}$ is the magnitude of the GAF and $\theta=\angle\left|G A F\left(\frac{\omega \bar{c}}{2 V}\right)\right|$ is the angle between in-phase and out-of-phase components of the GAF, in radians.

With these time-dependent representations, it can be shown that the parametric trace of these functions becomes a rotated ellipsoid of the form,

$$
\left(\frac{y}{B}\right)^{2}-2 \cos \theta\left(\frac{y}{B}\right)\left(\frac{x}{A}\right)+\left(\frac{x}{A}\right)^{2}=\sin ^{2} \theta
$$

\section{Relationship between Experimental and Analytical Models}

Based on the steady model shown in Eq. 9 and the corresponding GAF model in Eq. 18, the following relationships connect experimental and analytical models. These terms define first-order, linear, harmonic response typically studied in forced-oscillation experiments.

Table 1. Steady Harmonic Model, In-Phase \& Out-of-Phase Terms.

\begin{tabular}{|c|c|c|}
\hline & Steady Model & GAF Model \\
\hline In-phase term & $\bar{C}_{a_{\beta}} \phi_{A} \sin (\omega t)$ & $B \cos (\theta) \sin (\omega t)$ \\
\hline Out-of-phase term & $\bar{C}_{a_{p}} \phi_{A} k \cos (\omega t)$ & $B \sin (\theta) \cos (\omega t)$ \\
\hline In-phase coefficient & $\bar{C}_{a_{\beta}}$ & $\frac{B}{A} \cos (\theta)$ \\
\hline Out-of-phase coefficient & $\bar{C}_{a_{p}}$ & $\frac{B}{A k} \sin (\theta)$ \\
\hline
\end{tabular}

The out-of-phase coefficient, $\bar{C}_{a_{p}}$, provides an estimate of aircraft damping assuming no frequency dependence or unsteady behavior is present. This coefficient can be expanded based on a conventional linear aerodynamic model $^{14}$ to highlight the relationship between the out-of-phase coefficient and the steady-flow damping term. As shown below in Eq. 21 for roll damping, the out-of-phase coefficient is equivalent to the damping when the $\dot{\beta}$ term or its unsteady equivalent, ${ }^{19}$ shown in Eqs. (10) and (11), is relatively small. 


$$
\bar{C}_{\ell p}=C_{\ell p}+C_{\ell \dot{\beta}} \sin \alpha
$$

\section{E. NASTRAN Roll Oscillation Model of the Sting Mounted GTM}

In an attempt to understand the structural dynamics encountered during wind-tunnel testing, a finite-element structural model was built of the sting fixture used to mount the GTM wind tunnel model for roll oscillation testing. The finite element model consists of a combination of the mass of the WT model and rigid-body structure supporting the aerodynamic lifting surface of the WT model already described earlier. The aerodynamic balance is inside the GTM WT model and is connected to the sting. As can be seen in Fig. 5, the sting is positioned at the bottom of the WT model and its opposite end extends at about eye level to the posts structure standing on the

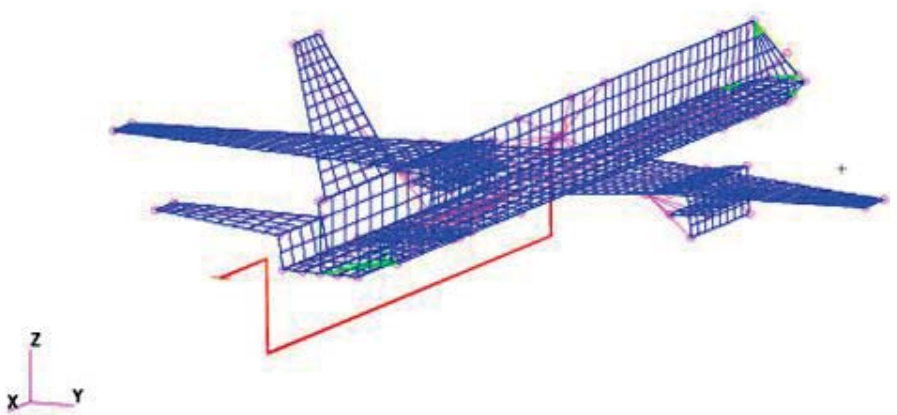

Figure 5. The finite element model and aero paneling of the GTM wind-tunnel model mounted on sting used for rolloscillation testing to obtain force and moment measurements. The FEM of the sting structure is shown in red.

$14 \times 22$ Wind Tunnel floor. However, the modeling of the sting can be simplified in recognition of the fact that the vertical post structure is very stiff. For the purposes of this study, the finite-element model of the sting was terminated to ground with the "T" structure at the post location. The available sting and balance drawings provided the geometry, as well as, physical and material property information for this finite-element modeling, while the balance calibration report gave the spring stiffness data of the aerodynamic balance in the six degrees of freedom (DOF) directions. To understand the structural dynamics of the GTM WT model assembly, a vibration analysis was conducted with NASTRAN, which produced 13 frequencies and mode shapes; however, only the first three fundamental frequencies and mode shapes of the structure are shown in Figs. 6a-6c. According to the analysis, there

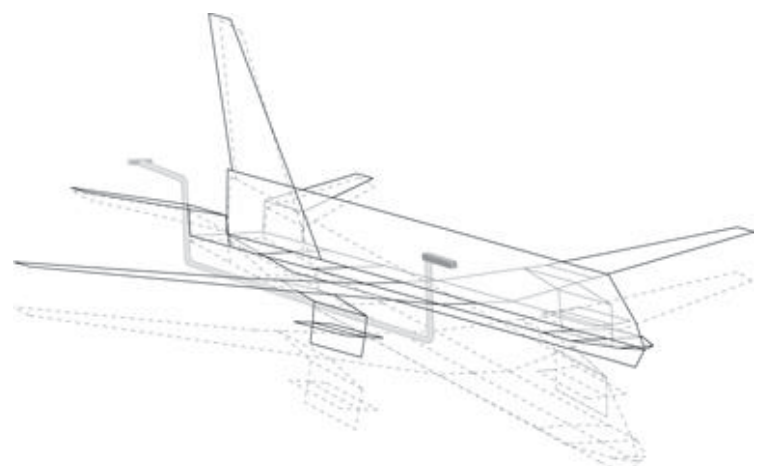

Figure 6a. First Longitudinal-Bending Mode, 4.93Hz.

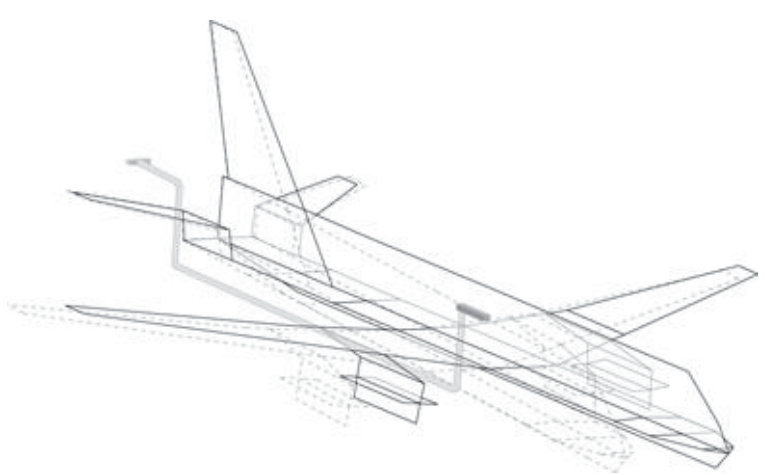

Figure 6b. First Lateral-Bending Mode, $6.37 \mathrm{~Hz}$.

is little more than $4 \mathrm{~Hz}$ separation between the highest roll oscillation frequency and the lowest vibration frequency.

The two lateral beams at the $\mathrm{T}$ part of the sting, as mentioned previously, are used here for a second purpose to provide the rolling motion to the sting, balance and also to the whole WTM assembly. The technique of Lagrange multiplier (see Ref. 7) is used to apply a sinusoidal torsion moment to a gridpoint between the two beams forcing oscillation on the WT model assembly in the roll direction as illustrated in Fig. 7. In the figure, the "T" represents the applied sinusoidal torque and the " $\theta$ " is the resulting angle of the two deformed beams to provide the sting rolling motion to generate the roll oscillations. 
Briefly, this computational operation for roll is achieved by adding one extra equation, associated with the Lagrange multiplier formulation, into the overall structural stiffness matrix, which is coupled to the two beam element's moment at the gridpoint in the roll direction. During NASTRAN processing, a column of a set of coefficients along with the coefficient of this extra equation is placed at the "right-hand-side" of the aeroelastic equations of motion allowing time dependent excitations to be inputted to the aeroelastic structure. Unfortunately, this procedure only allows force or moment excitations to be input,

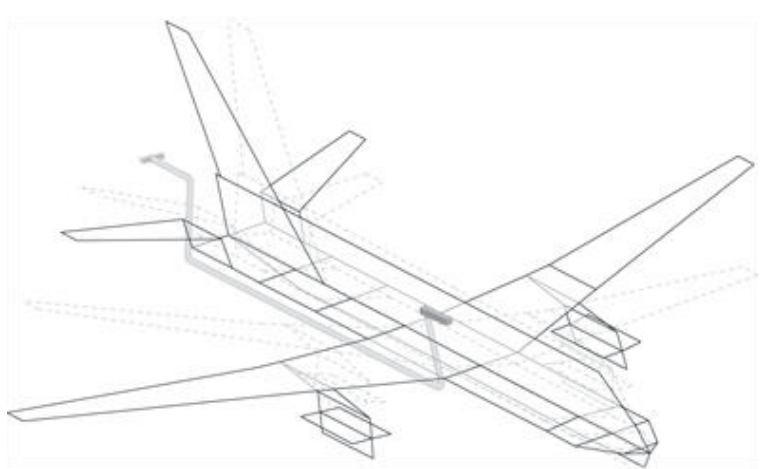

Figure 6c. First Roll-Torsion Mode, $50.1 \mathrm{~Hz}$. producing an unknown amount of gridpoint rotational deflection. However, through a "gain" parameter, DAREA, a desired gridpoint deflection can be adjusted by performing at least two NASTRAN "runs." One run is required to establish the existing gain value by looking at the deflection generated, then a second run is performed using an appropriately proportioned gain value to obtain the desired rotational deflection at the gridpoint and for the sting.

NASTRAN's Aeroelastic Response Analysis (see Ref. 7) simulates the roll motion in the frequency domain using solution 146. This approach is suited to solving these types of time-response problems since the predominate dynamics involve an aeroelastic system of equations including structural and frequency-dependent unsteady aerodynamics.

For this problem the sinusoidal time-history torque excitations are first converted to the frequency domain and processed along with the frequency-dependent unsteady aerodynamic loads that include the frequencies, mode shapes and the generalized mass of the finiteelement structure to produce a set of very large frequencydependent aeroelastic transfer functions. The products of each of these transfer functions and the transformed torque excitation produce the rolling-motion responses of the GTM WT assembly in the frequency domain. These frequency-dependent responses are then transformed back into the time-domain to obtain the time-history responses of the WT model assembly.

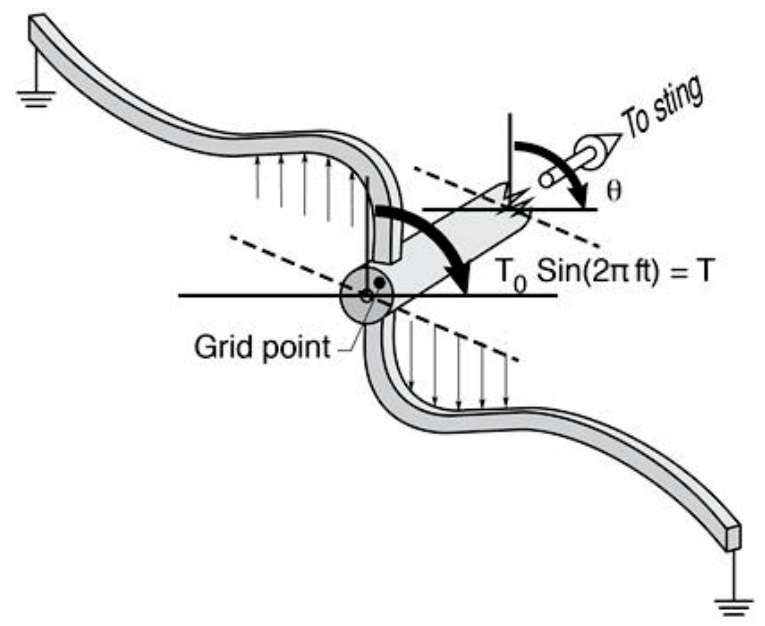

Figure 7. An illustration demonstrating the application of Lagrange multiplier technique of inputting sinusoidal torque excitations into the GTM WT model assembly with NASTRAN.

\section{Analytical and Experimental Comparisons}

Analytical and experimental results are comparable only at low angles of attack where linear aerodynamic behaviors occur. For the GTM model the linear region is below 10 degrees angle of attack where stall occurs. Tunnel measurements are presented over a large range of angle of attack to show the large variation in roll damping behavior and to clearly define the appropriate region for analysis.

\section{A. GAF Results}

Six GAFs, as a function of frequency, are shown in Fig. 8 representing the aerodynamic forces and moments caused by the roll motion. Ordinate values were removed on all plots in order to maintain proprietary agreements. The ratio roll moment to roll (angle) deflection increases linearly with frequency as expected; the slope of the function with circular frequency is proportional to the roll damping coefficient; and the 90 degrees phase angle of the coefficient shows as well the appropriate lag characteristic for damping. The GAF representing the ratio of the yaw moment to roll deflection provides a large yaw moment contribution especially at the higher frequencies. Potentially it appears the force produced by this yaw moment could excite lateral bending modes of the fuselage at 
the higher reduced frequencies. The remaining GAFs in this group provided relatively small force or moment contributions. The roll moment phase response as given by the GAF is consistant at the lower reduced frequency range at 90 degrees. This angle simplifies Eq. (20) to non-rotated form of the equation of an ellipse,

$$
\left(\frac{y}{B}\right)^{2}+\left(\frac{x}{A}\right)^{2}=1
$$

\section{B. Experimental Results}

Results of harmonic analysis performed on roll oscillatory data with amplitude of $20^{\circ}$ is presented in Fig. 9. These plots show in-phase and out-of-phase components against angle of attack at different frequencies and are plotted with the same scales. The range of frequencies chosen for the forcedoscillation test allows identification of aerodynamic transfer functions that can include unsteady aerodynamic behaviors for a rigid-body aircraft. Although the range of frequencies is driven by aircraft aerodynamics, the tests place demands on the balance and dynamic test rig structure that can excite structural mode responses. The plots indicate very limited frequency dependence (unsteady aerodynamic behavior) for the in-phase component.

Frequency dependence in the damping term or the out-ofphase component is present at $\alpha_{0}=12^{\circ}$. The unsteady behavior also appears in two regions: $24^{\circ} \leq \alpha_{0} \leq 30^{\circ}$ and for $\alpha_{0}>40^{\circ}$. Roll damping instabilities are present at $\alpha_{0}$ near $12^{\circ}$, $14^{\circ}$, and above $40^{\circ}$. For angles of attack below $10^{\circ}$ the aerodynamic behavior is very linear and steady. $R^{2}$ values are very close to 1 through $10^{\circ}$ angle of attack, indicating the linear aerodynamic model has explained virtually all the variation in the data. The region below $10^{\circ}$ angle of attack is the most appropriate region for comparisons with the doublet-lattice predictions provided in this study.

\section{Comparison of GAF and Experimental Results}

The GAF model presented in Fig. 8 shows a constant phase and linear variation of roll moment to roll angle with non-dimensional frequency, $k$. This is consistent with the wind tunnel inphase and out-of-phase results showing relatively constant values and no frequency dependence. Table 1 relationships show that the out-of-phase term is linearly proportional to $k$ consistent with the GAF term. The GAF value, converted to an out-of-phase component, is overplotted in Fig. 9 at low angle of attack to show the relationship with experimental data. This result is further confirmed by plotting the predicted time history at one test

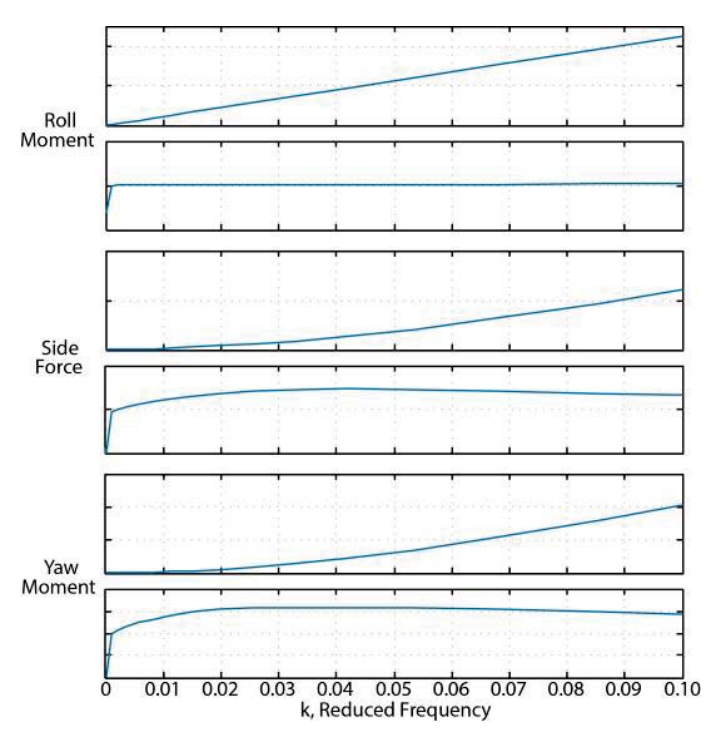

Figure 8. GAF frequency response plots for roll oscillation; generated by the NASTRAN doubletlattice code. 
condition, as shown in Fig. 10. Measured data sample rate was $250 \mathrm{~Hz}$ with a low-pass 100 $\mathrm{Hz}$ analog (anti-aliasing) filter.

\section{NASTRAN Roll Motion Simulation of the GTM Wind-Tunnel Model}

NASTRAN Aeroelastic Response Analysis was performed at the same test conditions as those used to simulate the windtunnel data presented in the generalized aerodynamic force coefficient section of this paper. The wind-tunnel results were obtained at a low speed of $92 \mathrm{feet} / \mathrm{second}$, at sea-level conditions and at a sinusoidal forcedoscillation frequency of $0.92 \mathrm{~Hz}$. The analysis performed with NASTRAN is linear, thus the results are only applicable at low angle of attack conditions.

Figure 11 shows time-history results of two NASTRAN analyses, one where the FEM masses were included in the analysis and the other without. The time response results of the roll and yaw moments demonstrate the effects of mass on the structural dynamic motion, especially where the rolling motion is starting and where it is stopping. The response plots to the left shows structural borne high-frequency dynamics at the points in time when excitation signal abruptly changes, from constant zero level to a sinusoidal form and later, abruptly from the sine oscillations back to a zero level. However, the response plots on the right do not show any of this type of behavior. This is consistent with the structural modes being at much higher frequencies than the test forced oscillation frequencies. In addition, at low alpha, the in-phase and out-of-phase coefficients show no frequency dependence or unsteady behaviors.

Figure 12 presents plots of roll and yaw moment versus roll angle. These plots are commonly used to graphically assess stability and control information. For the low alpha case without unsteady behavior, the y-axis intercept is in proportion to out-of-phase component (damping) and rotation of the ellipse reflects the in-phase component (stability). A distinct feature observed in the roll moment ellipsoid plots is that they are slightly rotated. This is consistent with the in-phase component measurements from the tunnel that show very small values for angles of attack below 10 degrees. Another observation, consistent for a conventional aircraft, is the relatively larger damping in roll as compared to yaw indicated by the much wider ellipses for roll. Also consistent with Fig. 11 time-history plots, the left plots show the same pronounced transients, when the analysis included the finite-element masses.

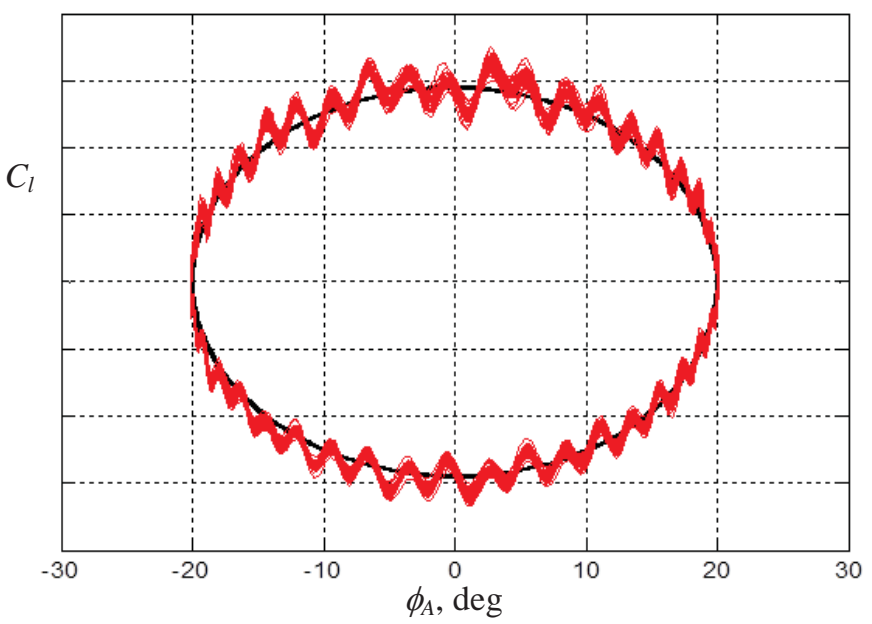

Figure 10. Doublet Lattice prediction and measurements of rolling moment versus roll angle during forced oscillations at $f=0.41 \mathrm{~Hz}, \alpha_{o}=0^{\circ}, \phi_{A}=20^{\circ}$.
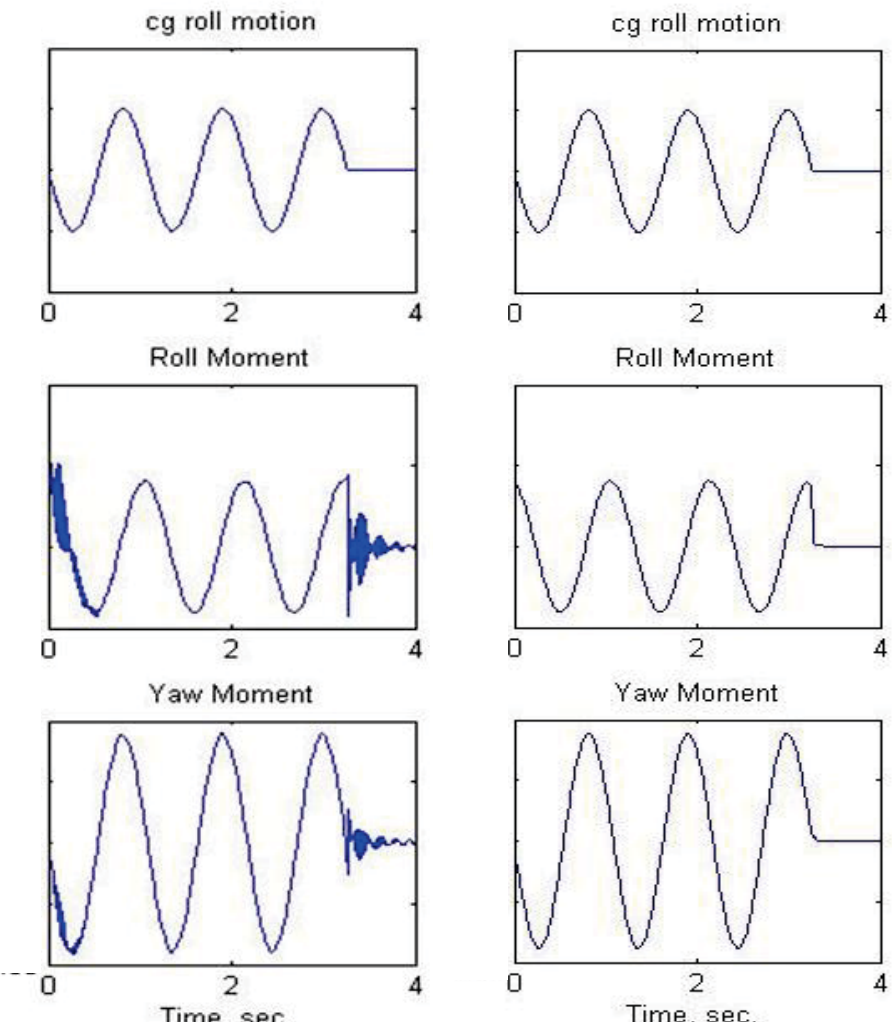

Figure 11. Roll oscillation time-history response plots of data generated by the NASTRAN Aeroelastic Response Analyses. Left column includes FEM masses; right column is without. 


\section{Concluding Remarks}

Since lifting surface modeling using doublet lattice was not available for analysis performed prior to this study, a main focus for this effort was devoted to creating unsteady aerodynamic boxes and a finite-element structural model of the GTM using the PATRAN, FLIGHT LOADS and NASTRAN software. This aeroelastic model was built from the "ground up" using an IGES geometry originally used to build a CFD model. Much of the work required simplifying a complex GTM moldline geometry into flatplate structures and aerodynamic panels for the aeroelastic model needed to generate unsteady generalized aerodynamic force coefficients by NASTRAN's doublet lattice code. With careful attention to moment reference center location, it was possible to calculate matching forces and moments to those obtained through experimentation.

A finite-element aeroelastic model of the 14x22 wind-tunnel model setup was also built and NASTRAN Aeroelastic Response Analysis was used to simulate the roll motion of the windtunnel model during testing. The simulation demonstrated the structural-dynamics effects on the aerodynamic balance data coming from the test rig during roll oscillation testing. NASTRAN analyses showed the rolling moment timeresponses with the same magnitude and phase observed in the wind tunnel dynamic tests. This initial study also indicates that structural mode responses for GTM dynamic tests are primarily occurring at $4 \mathrm{~Hz}$ and above. This suggests a threshold for including higher-order harmonics in aerodynamic models for GTM rigid-body aircraft dynamic studies. Additional studies will be required to further separate and identify the
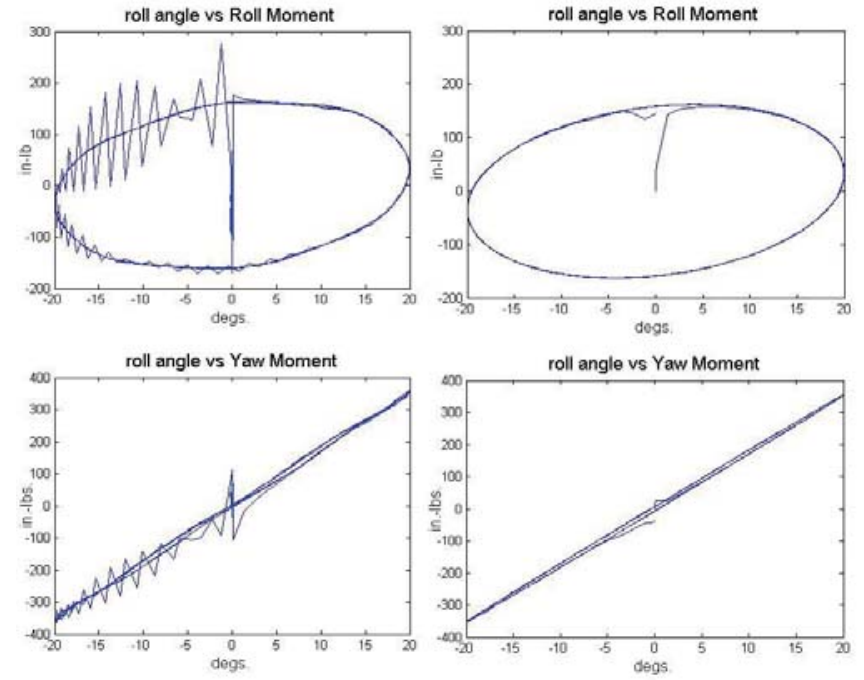

Figure 12. Roll oscillation plots showing NASTRAN predictions and measurements with left plots including structural masses and right plots without masses. source of continuous vibrations measured during steady harmonic forced-oscillations. Potential sources that can excite these vibrations can come from imperfect input sinusoids producing a range of additional input frequencies or imbalances in the mechanical forced-oscillation rig and model.

With appropriate assumptions, doublet lattice can be effective in deriving dynamic stability derivatives of transport configurations. Also NASTRAN Aeroelastic Response Analysis can be used to simulate the roll or other motions of the wind-tunnel model and test rig used during testing to give an early assessment, in terms of loads and dynamics, of a test fixture setup and design.

\section{Acknowledgments}

The authors extend their appreciation to the NASA Aviation Safety Program, Vehicle Systems Safety Project for the support provided.

\section{References}

${ }^{1}$ Belcastro, Christine M.; Foster, John V.: “Aircraft Loss-of-Control Accident Analysis," AIAA Guidance, Navigation, and Control Conference, August 2010, Toronto, Canada, AIAA 2010-8004.

${ }^{2}$ Foster, John V., et al: "Dynamics Modeling and Simulation of Large Transport Airplanes in Upset Conditions," AIAA Guidance, Navigation, and Control Conference, August 2005, San Francisco, California, AIAA 2005-5933.

${ }^{3}$ Thompson, Joseph R.; Frink, Neal T.; Murphy, Patrick C.: "Guidelines for Computing Longitudinal Dynamic Stability Characteristics of a Subsonic Transport," AIAA Applied Aerodynamics Conference, June 2010, Chicago, Illinois, AIAA 20104819.

${ }^{4}$ Morelli, Eugene A.: "Flight Test Maneuvers for Efficient Aerodynamic Modeling," AIAA Atmospheric Flight Mechanics Conference, August 2011, Portland, Oregon, AIAA 2011-6672.

${ }^{5}$ Murphy, Patrick C.; Klein, Vladislav.: "Transport Aircraft System Identification Using Roll and Yaw Oscillatory Wind Tunnel Data," AIAA Atmospheric Flight Mechanics Conference, August 2010, Toronto, Canada, AIAA $2010-8122$. 
${ }^{6}$ Murphy, Patrick C.; Klein, Vladislav; Frink; Neal T.; Vicroy, Dan D.:'System Identification Applied to Dynamic CFD Simulation and Wind Tunnel Data," AIAA Atmospheric Flight Mechanics Conference, August 2011, Portland, Oregon, AIAA 2011-6522.

${ }^{7}$ Rodden, William R.; Johnson, Erwin: “MSC/NASTRAN Aeroelastic Analysis, User’s Guide,” v68, 1994.

${ }^{8}$ DMAP Programmer's Guide, Nastran 2012.2, MSC.Software Corporation, 2012

${ }^{9}$ Rodden, William R.; Giesing, J, P.: “Application of Oscillatory Aerodynamic Theory to the Estimation of Dynamic Stability Derivatives, Journal of Aircraft, Vol.8, pp. 272-275, 1970.

${ }^{10}$ Murphy, Patrick C.; Klein, Vladislav; Frink, Neal T.: "Unsteady Aerodynamic Modeling in Roll for the NASA Generic Transport Model," AIAA Atmospheric Flight Mechanics Conference, August 2012, Minneapolis, Minnesota, AIAA $2012-4652$.

${ }^{11}$ Murphy, Patrick C.; Klein Vladislav: "Validation of Methodology for Estimation Aircraft Unsteady Aerodynamic Parameters from Dynamic Wind Tunnel Tests," AIAA Atmospheric Flight Mechanics Conference, August 2003, Austin, Texas, AIAA 2003-5397.

${ }^{12}$ Tobak, M., Schiff, L.: "On the Formulation of the Aerodynamic Characteristics in Aircraft Dynamics," NASA TR R-456, 1976.

${ }^{13}$ Klein, Vladislav; Norderer, Keith D.: "Modeling of Aircraft Unsteady Aerodynamic Characteristics. Part I - Postulated Models," NASA TM 109120, May 1994

${ }^{14}$ Klein, Vladislav; Murphy, Patrick C.; Szyba, Nathan M.: “Analysis of Wind Tunnel Lateral Oscillatory Data of the F-16XL Aircraft," NASA/TM-2004-213246, 2004. 